\title{
28 Research Square \\ Protective Effect of Ulinastatin on Acute Lung Injury in Diabetic Sepsis Rats
}

\section{Yufeng Zou}

Zhongnan Hospital of Wuhan University

\section{Hu Chen}

Maternal and Child Health Care Hospital of Bao'an

\section{Meng-Yu Li}

Zhongnan Hospital of Wuhan University

\section{Lijuan Tang}

Zhongnan Hospital of Wuhan University

\section{Zhe Jin}

Zhongnan Hospital of Wuhan University

Kai Chen ( $\square$ chenkg1203@126.com )

Zhongnan Hospital of Wuhan University

\section{Research Article}

Keywords: diabetes mellitus, Sepsis, Acute lung injury, ulinastatin

Posted Date: October 27th, 2021

DOl: https://doi.org/10.21203/rs.3.rs-882532/v1

License: (c) (1) This work is licensed under a Creative Commons Attribution 4.0 International License. Read Full License 


\section{Abstract}

\section{Objective}

To establish a rat model of diabetic sepsis, to observe the protective effect of ulinastatin on acute lung injury in diabetic sepsis rats, and to explore the possible mechanism from the aspects of inflammatory response, oxidative stress, hypoxia-inducible factor- 1 a expression and pulmonary microvascular permeability.

\section{Methods}

A total of 50 SPF adult male SD rats were randomly selected, 38 were fed with high fat diet, and the remaining 12 were fed with ordinary diet. Feed five weeks later, the fatty group according to $30 \mathrm{mg} / \mathrm{kg}$ body weight, abdominal single injection of STZ, three days after injection, in a random blood glucose or greater tendency for $16.7 \mathrm{~L}$ for type 2 diabetes mellitus rats building success, take 36 ChengMo rats were randomly divided into four groups, each group of 12, namely D group of type 2 diabetes, the DS group of type 2 diabetes mellitus and sepsis, Group U ulinastatin pretreatment group. Twelve ordinary feed group were selected as $\mathrm{C}$ group, namely blank control group. After successful modeling of type 2 diabetic rats, DS group and $U$ group were injected with endotoxin (LPS) at $5 \mathrm{mg} / \mathrm{kg}$ via tail vein to construct diabetic sepsis lung injury rat model. Group U was injected with ulinastatin $100 \mathrm{kU} / \mathrm{kg}$ caudal vein one hour before LPS. 4h later (4 rats in each group were injected with $2 \mathrm{~mL} \mathrm{1 \%}$ Evans blue solution through tail vein half an hour before execution), blood was collected and lung tissues were removed. HE staining was used to observe the pathological changes of lung tissues. The wet/dry ratio (W/D) of lung tissue was determined. Serum IL-1 $\beta$, IL-18 and TNF-A were detected by ELISA. The contents of malondialdehyde (MDA) and superoxide dismutase (SOD) in serum were detected according to the kit instruction. Western blot was used to detect the hypoxia-inducible factor-1 a (HIF-1a) protein in renal tissue. The expression of TLR4mRNA in lung tissues was detected by rt-pcr. Evans blue staining was used to detect pulmonary microvascular permeability.

Results

Compared with the control group, the lung interstitium in group D was thickened to a certain extent, with a small amount of inflammatory cell infiltration and a little exudate in some alveolar cavities. Compared with D group, DS group had more serious damage, with obvious pulmonary interstitial hyperemia and edema, a large amount of exudation in alveolar cavity, significantly increased inflammatory cells, necrosis and swelling of alveolar epithelial cells. In group $\mathrm{U}$, there were more inflammatory cells in the interstitium and part of alveolar cavities, epithelial cells were exfoliated occasionally in the lumen, and the interstitium widened and the loss was less than that in group DS. Compared with group $C$, the wet/dry weight ratio (W/D), serum IL-1 $\beta$, IL-18 and TNF-A contents, hiF-1 a protein expression, TLR4 mRNA expression and pulmonary microvascular permeability of rats in other groups were significantly increased $(P<0.01$ or $P<0.05)$. The content of MDA and SOD activity in serum decreased $(P<0.01)$. Compared with group $D$, the wet/dry weight ratio (W/D), serum IL-1 $\beta$, IL-18 and TNF-A contents, hiF-1 a protein 
expression, TLR4 mRNA expression and pulmonary microvascular permeability in DS group and $\mathrm{U}$ group were significantly increased $(P<0.01$ or $P<0.05)$. The content of MDA in serum was increased $(P<0.01$ or $P<0.05)$, and the activity of SOD in DS group was decreased $(P<0.01)$.; Compared with DS group, the wet/dry weight ratio (W/D), serum IL-1 $\beta$, IL-18, TNF-A contents, hiF-1 a protein expression, TLR4 mRNA expression, and pulmonary microvascular permeability of rats in GROUP $U$ were significantly decreased $(P<0.01$ or $P<0.05)$. The content of MDA in serum decreased $(P<0.01)$, and the activity of SOD increased $(P<0.01)$.

\section{Conclusion}

Ulinastatin can effectively reduce acute lung injury induced by diabetic sepsis in rats, and its mechanism may be related to inhibiting inflammatory response, reducing oxidative stress, regulating hypoxia response pathway and improving pulmonary microvascular permeability.

\section{Background}

Diabetes has become the biggest health killer in China, and the age of onset is getting younger and younger. However, there is a serious lack of diabetes hazard and vigilance in China. Studies have shown that long-term hyperglycemia leads to a series of biochemical metabolism and defense dysfunction. When patients suffer from surgical trauma, artificial assisted breathing through endotracheal intubation, long-term bed rest or coma, it is easy to lead to secondary pulmonary infection in patients with diabetes, among which gram-negative bacteria account for $77.35 \%{ }^{[1,2]}$. Therefore, the study and discussion of the possible mechanism of respiratory tract infection in diabetic patients and the effective treatment plan to reduce their lung injury will provide an important theoretical basis for the prevention and treatment of diabetic lung infection. At present, it has been confirmed that the occurrence of inflammatory response in diabetic patients can be realized through toll-like receptor 4 (TLR4) pattern recognition receptor $[3,4]$. Studies on the expression changes of TLR4 in the lung tissues of diabetic rats and the reactivity of TLR4 to lipopolysaccharide showed that TLR4 could not play the normal immune recognition function under immune stress conditions, leading to the decrease of the immune defense ability of the body and the susceptibility to lung infection. Studies have shown that clinically relevant lung tissue inflammation is less prevalent in TLR4 gene deletion animals ${ }^{[5]}$, where the Toll/ interleukin-1 receptor binding region induces interferon $\beta$ (TRIF) pathway to play an important role in the inflammatory response induced by endotoxin-induced lung injury ${ }^{[6]}$. Activation of TLR4 can promote the production and release of a large number of inflammatory factors, and further mediate the production of TNF- $a$, il- $1 \beta$, il-18 and other inflammatory factors. The "cascade amplification" effect of inflammation damages pulmonary vascular

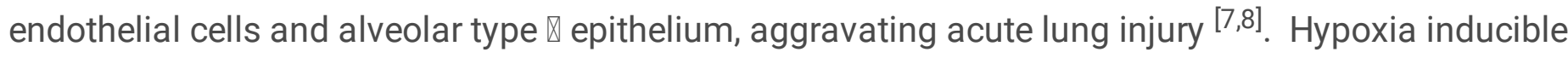
factor $1 \mathrm{a}(\mathrm{HIF-1a})$ is a key factor specifically mediating hypoxia response in the regulation of oxygen balance in cells. Most cells express HIF-1a in response to hypoxia. As an intermediary factor in physiological and pathological responses of hypoxia adaptation, hypoxia-inducible factor- 1 is involved in the transcription and expression of many related genes ${ }^{[9]}$. Ulilnastatin (UTI) is a broad-spectrum 
protease inhibitor that inhibits the release of a variety of proteases, lipid hydrolases, glucose hydrolases and inflammatory factors induced by adverse stimuli ${ }^{[10,11]}$. In recent years, there are data shows UTI inhibiting release of inflammatory mediators, prevention of cytokine cascade, inhibition of leucocyte excessive activation, block the vicious circle between cytokines and white blood cells, in sepsis, DIC, multiple organ failure and so on systemic inflammatory disease of the lung protection ${ }^{[10-12]}$, but its detailed mechanism is still not very clear. In view of the previous studies and the above theories, there are few studies on the cell signaling pathway of diabetic lung infection, and the protective effect of ulinastatin on IPS-induced acute lung injury in diabetic rats and its related mechanisms have not been reported. Therefore, this study intended to establish a model of LPS induced acute lung injury in diabetic rats, and to observe the protective effect of ulinastatin on the lung of diabetic sepsis rats by intravenous injection of ulinastatin for pretreatment. To explore the possible mechanism of ulinastatin's lung protection in diabetic sepsis rats from the aspects of inhibiting inflammatory response, anti-oxidative stress, regulating hypoxia response pathway and improving pulmonary microvascular permeability, and provide theoretical basis for the prevention and treatment of diabetic pulmonary infection and the development of new gene therapy measures by regulating related signaling pathways.

\section{Materials And Methods}

Material

Fifty healthy male Sprague Dawley (SD) SPF rats in the same batch, weighing 180 220g, provided by The Animal Experimental Center of Wuhan University [Certificate No. : SCXK(E)2009-0025], License No. : $\operatorname{SCXK}(E) 2008-0004$. Feeding environment: photoperiod of 12 hours, temperature $21-25^{\circ} \mathrm{C}$, humidity $50 \%-60 \%$. Two weeks before modeling, routine feeding, the night before fasting without drinking. All experimental procedures were approved by the Animal Ethics Committee of Wuhan University, China, and were performed in accordance with the National Institutes of Health "Guidelines for the Care and Use of Laboratory Animals". This study was approved by the animal ethics committee at the Zhongnan Hospital and Research Centre, Hubei, China and performed at animal experimental center of Wuhan University, China. The study was carried out in compliance with the ARRIVE guidelines.

\section{Experimental groups}

38 were randomly selected and fed with high-fat feed, and the remaining 12 were fed with ordinary feed. Animal feed was mainly prepared by referring to the method used by Vaneker $M$ et al ${ }^{[13]}$. STZ powder was dissolved in citric acid buffer and mixed into a solution with a concentration of $1 \%$ and $\mathrm{PH}$ value of 4.5 for streptozotocin solution for diabetes modeling. After 5 weeks of feeding, the high-fat group received a single intraperitoneal injection of STZ at the weight of $30 \mathrm{mg} / \mathrm{kg}$. After 3 days of injection, the model of type 2 diabetes rats with random blood glucose $\geq 16.7 \mathrm{mmol} / \mathrm{L}$ was successfully established. 36 model rats were randomly divided into 3 groups with 12 rats in each group by numerical random method, namely group $D$ type 2 diabetes group and GROUP DS type 2 diabetes combined with sepsis group. $U$ group ulinastatin treatment group. Twelve ordinary feed group were selected as $\mathrm{C}$ group, namely blank 
control group. Lipopolysaccharides (LPS) is a type of lipopolysaccharide, which exists in the cell wall of gram-negative bacteria, and gram-negative bacteria infection is one of the main pathogenic factors of ALI [5]. After the successful modeling of type 2 diabetic rats, DS group and $U$ group were injected with endotoxin (LPS) at a dose of $5 \mathrm{mg} / \mathrm{kg}$ via tail vein to construct diabetic sepsis lung injury rat model. Group U was given ulinastatin $100 \mathrm{kU} / \mathrm{kg} 1 \mathrm{~h}$ before LPS, diluted to $1 \mathrm{~mL}$ of normal saline and slowly injected into tail vein $(5 \mathrm{~min})$. Four rats in each group were anesthetized with $2 \%$ pentobarbital sodium $(2 \mathrm{~mL} / \mathrm{kg}, \mathrm{IP})$. The rats were decapitated and sacrificed. Blood was collected and lung tissues were taken out for frozen use. All experimental procedures were approved by the Animal Ethics Committee of Wuhan University, China, and were performed in accordance with the National Institutes of Health "Guidelines for the Care and Use of Laboratory Animals". This study was approved by the animal ethics committee at the Zhongnan Hospital and Research Centre, Hubei, China and performed at animal experimental center of Wuhan University, China.

HE staining was used to observe the pathological changes of lung tissues:

The lungs of rats were used.After fixation with $4 \%$ paraformaldehyde for $24 \mathrm{~h}$, paraffin embedded and cut into $4 \mu \mathrm{m}$ slices, dried and dewaxed. HE staining was performed according to the instructions of the $\mathrm{HE}$ staining kit, and the histological changes were observed at $400 \mathrm{x}$ microscope.

Wet/dry weight ratio of lung tissue (W/D) :

After the rats were sacrificed, the lung was removed through thorax and the middle lobe of the right lung was separated for W/D ratio determination. Remove connective tissue, dry the water with filter paper and put it on an electronic balance to weigh and record the wet weight, dry it in an oven at $80^{\circ} \mathrm{C}$ for $72 \mathrm{~h}$ to constant weight, weigh the dry weight and calculate the wet-dry weight ratio (W/D).

Serum LEVELS of IL-1 $\beta$, IL-10 and TNF-A were detected by ELISA:

The contents of IL-1 $1 \beta$, IL-10 and TNF-A in the supernatant of culture medium were detected according to the instructions of ELISA kit.

Determination of serum oxidative stress index:

Determination of MDA content in serum: Thiobarbituric acid (TBA) was used to determine MDA content in serum of rats. It is mainly based on the following principle: when the ambient temperature is high and acidic, MDA can react with TBA to produce red mda-tba adducts. The maximum absorption wavelength of this substance is $535 \mathrm{~nm}$, which can be detected by colorimetric method.

Determination of SOD activity in serum: The SOD activity in serum of rats was determined by xanthine oxidase method.

Western blot analysis of hiF-1 a expression in lung tissue: 
Nuclear proteins were extracted using the protein extraction reagent provided by the nuclear protein and cytoplasmic protein extraction kit. The extracted nucleoprotein was stored at $-70^{\circ} \mathrm{C}$ for later use. $50 \mu \mathrm{g}$ nucleoprotein was added to each sample for SDS-PAGE protein electrophoresis. The isolated nucleoprotein was then transferred to PVDF membrane, which was treated in blocking solution (5\% skim milk powder dissolved in PBS buffer $-0.05 \%$ Tween 20 ) at room temperature for $1 \mathrm{~h}$ to block non-specific binding. Then the primary antibody (anti- $\beta$-Arrestin-2, anti- $\beta$-Actin) was added and incubated at $4{ }^{\circ} \mathrm{C}$ overnight. The filter membrane was rinsed with 1×TBST 3-4 times, 10 min each time, and the secondary antibody HRP-Sheep anti-mouse IgG was added and incubated at room temperature gently for $1 \mathrm{~h}$. The membrane was rinsed with $1 \times$ TBST for 3-4 times, 10 min each time, and the enhanced chemiluminescence reaction was performed at last. Optical density scanning for semi-quantitative analysis of developing tape.

Detection of TLR4 mRNA expression level in lung tissues by RT-PCR:

According to the cDNA sequence of RAT M5 receptor in GeneBank, Primer software was used to design and amplify the TLR4 gene sequence. The primers were synthesized by Invitrogen. Total RNA was extracted from lung tissues and cell lines using miRNeasy kits, and RNA quality and concentration were measured by Nano-DROP spectrophotometer. The mRNA was reversely transcribed into cDNA using the PrimeScriptRT Master Mix Kit, and the mRNA was amplified and detected according to the INSTRUCTIONS of the TB Green PCR Kit. The circulation conditions of TLR4 were 40 cycles at $94{ }^{\circ} \mathrm{C}$ for $20 \mathrm{~s}$ and $60^{\circ} \mathrm{C}$ for $60 \mathrm{~s}$, standardized by glyceraldehyde 3-phosphate dehydrogenase (GAPDH). The analysis was repeated three times for each sample. $2-\triangle \triangle \mathrm{Ct}$ values were calculated according to the cyclic threshold $\mathrm{Ct}$ values of each group as the relative expression of TLR4. All primers were synthesized by Shanghai Shenggong Biological Co., LTD., and the primer information was as follows: TLR4 sequence (amplification fragment: 215bp) upstream primer: 5'- GCTCTGGCATCATCTTCATTGT-3'; Downstream primer: 5'- TGTTGCTTCTTGTTCTTCCTCT- 3'; Upstream primer of b-actin sequence (110bp amplification fragment) : 5'- CGTTGACATCCGTAAAGACCTC-3'; Downstream primer: 5'- TAGGAGCCAGGGCAGTAATCT$3^{\prime}$.

Pulmonary microvascular permeability test:

Four rats in each group were taken and injected 1\% Evans blue solution (EB) $2 \mathrm{~mL} / \mathrm{kg}$ through tail vein half an hour before death. After death, the right ventricle was pierced with no. 5 needle, and the left ventricle was cut open. The pulmonary vessels were rinsed with low-concentration heparin solution $(500 \mathrm{~mL}$ of normal saline and $0.5 \%$ heparin $1 \mathrm{~mL}$ ) until the left ventricle flushing solution was clarified. Take part of the left lung tissue, rinsed three times in $0.9 \%$ salt water ice, filter paper surface moisture blot, weigh wet quality, cut into small blocks, lung tissue soaked in formamide solution, in constant temperature water bath extraction in the groove $24 \mathrm{~h}$, centrifugal, take the supernatant, using ultraviolet spectrophotometer colorimetric method (wavelength of $620 \mathrm{~nm}$ ) absorbance of leach liquor, EB content was measured on a standard curve, and pulmonary microvascular permeability was measured as EB $\mu \mathrm{g}$ per gram of lung tissue. 
Statistical analysis

All data are presented as mean (SD). Statistical analyses were performed using SPSS (version 13.01S; Beijing Stats Data Mining Co. Ltd, Beijing, China). ANOVA was used to compare the mean of each group, and LSD was used for pairwise comparison. $P<0.05$ was considered as significant difference.

\section{Results}

Pathological changes of lung tissues in each group

In the normal control group, the lung tissue structure was intact, the alveolar wall was thin and regular without collapse, the pulmonary interstitium was not dilated and engorged, the alveolar cavity was not infiltrated by inflammatory cells and red blood cells, and the bronchial mucosa was intact without epithelial cells falling off. In group D, lung interstitium was thickened to a certain extent, with a small amount of inflammatory cell infiltration and a little exudate in some alveolar cavities. In the DS group, the lung tissue structure was deformed, the alveolar wall collapsed, the lung interstitium widened, and presented atelectasis. A large number of inflammatory cells infiltrated and red blood cells were observed in the lung interstitium and alveoli, exfoliated epithelial cells were observed in the bronchi, and a large number of telangiectasia were observed, and severe lung bubble cavities were reduced or even disappeared. The pathological changes of lung tissue in group $U$ were lighter than that in group DS, with capillary congestion, some red blood cells in capillaries, more inflammatory cells in interstitium and part of alveolar lumen, occasional exfoliated epithelial cells in the lumen, and interstitium widened. As shown in figure 1 .

Comparison of wet/dry weight ratio (W/D) of lung tissue in each group

Compared with $\mathrm{C}$ group, the W/D of DS group and $\mathrm{U}$ group was significantly higher $(P<0.01)$. Compared with $\mathrm{D}$ group, THE W/D in DS group and $\mathrm{U}$ group was significantly higher $(P<0.01)$. Compared with DS group, W/D of lung tissue in $\mathrm{U}$ group was significantly decreased $(P<0.05)$. As shown in table 1 .

Comparison of serum IL-1 $\beta$, IL-18 and TNF-A contents in each group

Compared with group $C$, serum IL-1 $\beta, \mathrm{IL}-18$ and TNF-A in other groups were increased $(P<0.01)$;

Compared with D group, serum IL-1 $\beta$, IL-18 and TNF-A were increased in DS group and U group $(P<0.01$ or $P<0.05)$. Compared with DS group, serum IL-1 $\beta$, IL-18 and TNF-A in U group were decreased $(P<0.01$ or $P<0.05)$. As shown in table 2.

Comparison of MDA content and SOD activity in serum of rats

Compared with group $C$, the content of MDA in serum in other groups was increased $(P<0.01)$, and the activity of SOD was decreased $(P<0.01)$. Compared with $\mathrm{D}$ group, MDA content in DS group and $\mathrm{U}$ group was increased $(P<0.01$ or $P<0.05)$, SOD activity in DS group was decreased $(P<0.01)$. Compared with DS 
group, serum MDA content in U group was decreased $(P<0.01)$, and SOD activity was increased $(P<0.01)$. As shown in table 3 .

Comparison of hiF-1 a protein expression in lung tissues of rats in each group

Compared with group C, the expression of HIF-1 a protein in other groups was significantly increased $(\mathrm{P}<0.01$ or $\mathrm{P}<0.05)$; Compared with $\mathrm{D}$ group, the expression of HIF-1a protein in $\mathrm{DS}$ group and $\mathrm{U}$ group was significantly increased ( $P<0.01$ or $P<0.05$ ); Compared with DS group, the expression of HIF-1a protein in lung tissue of $U$ group was significantly decreased $(P<0.05)$. As shown in figure 2.

Comparison of TLR4 mRNA expression in lung tissues of rats

Compared with group $\mathrm{C}$, the expression of TLR4 mRNA in other groups was significantly increased $(P<$ 0.01). Compared with D group, the expression of TLR4 mRNA in DS group and $U$ group was significantly increased $(P<0.01)$. Compared with DS group, TLR4 mRNA expression in lung tissue of $U$ group was significantly decreased $(P<0.01)$. As shown in table 4 .

Comparison of pulmonary microvascular permeability in each group

Compared with group $C$, the content of Evans blue (EB) in lung tissue of group D showed an increasing trend but no significant difference $(P>0.05)$, while the content of Evans blue (EB) in lung tissue of group DS and group $U$ was significantly increased $(P<0.01)$. Compared with $\mathrm{D}$ group, EB content in $\mathrm{DS}$ group and $U$ group was significantly increased $(P<0.01)$. Compared with $\mathrm{DS}$ group, EB content in lung tissue of $\mathrm{U}$ group was significantly decreased $(P<0.01)$. As shown in table 5

\section{Discussion}

As a common disease with frequent occurrence, the prevalence and incidence of diabetes in China have been increasing year by year in recent years. And after suffering from diabetes, due to long-term hyperglycemia, can lead to a variety of complications, especially infection. When patients with diabetes experience surgical trauma again, artificial assisted breathing with tracheal intubation, long-term bed rest or coma, patients with diabetes are prone to secondary pulmonary infection and may further lead to acute lung injury ${ }^{[1,2]}$.

Ulinastatin, purified from human urine, is a glycoprotein with a molecular weight of $67 \mathrm{kDa}$. As a protease inhibitor, it can effectively inhibit a variety of enzymes and inflammatory reactions caused by adverse stimulation. In addition, it can improve microcirculation by clearing excessive oxygen free radicals, stabilizing lysosomal membrane and preventing the release of inflammatory factors. Studies have shown that ulinastatin can inhibit the release of inflammatory mediators, prevent the cascade of cytokines, inhibit the excessive activation of leukocytes, and block the vicious cycle between cytokines and leukocytes. Ulinastatin has lung protective effects in systemic inflammatory diseases such as sepsis, DIC and multiple organ failure ${ }^{[11-13]}$. 
In this study, high-fat diet supplemented with single-dose intraperitoneal injection of streptozotocin (STZ) was used to construct the T2DM rat model. The characteristic of this approach is that low doses of STZ can destroy the function of rat islet $\beta$ cells, while feeding with high fat diet can reduce the sensitivity of peripheral tissues to insulin, thus forming a similar T2DM model. In this study, a total of 38 rats were enrolled for modeling, of which 1 died. Finally, 36 rats with successful modeling were selected for the next stage of animal model construction.

The main manifestations of acute lung injury caused by sepsis are as follows: Alveolar epithelium and extensively damaged alveolar capillary wall accompanied by obvious inflammatory edema, alveolar walls are broken, the thickening of alveolar interval, and red blood cells and inflammatory cells into the alveolar cavities, severe damaged alveolar epithelial cells, damaged alveolar surface active substances, with the progress of the course, part of the lung atelectasis can occur.

In this study, the lung interstitium of rats in group D was thickened to a certain extent, with a small amount of inflammatory cell infiltration and a little exudate in some alveolar cavities, which might be caused by lung microvascular injury caused by long-term hyperglycemia. DS group rats significantly higher, W/D structure deformation, lung tissue alveolar walls collapse, pulmonary interstitial broadening, atelectasis, pulmonary interstitial and alveolar we see a lot of inflammatory cells infiltration and red blood cells, endobronchial visible loss of epithelial cells, numerous capillaries expansion at the same time, serious lung bubble cavity reduced or even disappeared, These results indicated that the rat model of diabetic sepsis with acute lung injury was successfully constructed. W/D in group $U$ was lower than that in group DS, and there were more inflammatory cells in the interstitial and part of alveolar cavities, exfoliated epithelial cells were occasionally found in the lumen, and the interstitial widened, and the pathological damage of lung tissue was lighter than that in group DS, suggesting that ulinastatin can alleviate acute lung injury in diabetic sepsis rats to a certain extent.

For patients with diabetes, pathophysiological changes such as decreased oxygen carrying capacity of red blood cells, increased stress level and thickened basement membrane of microvessels caused by long-term hyperglycemia and metabolic disorders can induce infection and lead to sepsis, and the degree of hyperglycemia can also be aggravated during the onset of sepsis. At present, it has been confirmed that the occurrence of inflammatory response in diabetes patients can be realized through toll-like receptor 4 (TLR4) pattern recognition receptor ${ }^{[14,15]}$. In this study, the expression of TLR4 in group D was significantly higher than that in the control group, which was consistent with previous studies. Studies on the expression changes of TLR4 in the lung tissues of diabetic rats and the reactivity of TLR4 to lipopolysaccharide showed that TLR4 could not play the normal immune recognition function under immune stress conditions, leading to the decrease of the immune defense ability of the body and the susceptibility to lung infection. In this study, after IPS-induced acute lung injury, TLR4 expression in DS group and $U$ group was significantly increased compared with that in $D$ group, indicating further activation of TLR4, while TLR4 expression in U group was significantly decreased compared with that in DS group after ulinastatin pretreatment, indicating that ulinastatin can inhibit TLR4 activation to a certain extent. 
Studies have shown that clinically relevant lung tissue inflammation is less prevalent in TLR4 gene deletion animals ${ }^{[5]}$, where the Toll/ interleukin-1 receptor binding region induces interferon $\beta$ (TRIF) pathway to play an important role in the inflammatory response induced by endotoxin-induced lung injury [6].

A large number of previous studies have shown that the serum levels of TNF-a, IL-10, IL-1, IL-6 and other inflammatory factors can be significantly reduced after the use of UTI, and the inflammatory response can be significantly reduced, and the anti-inflammatory effect of UTI is obvious [16-19]. In particular, serum TNF-a levels will be very low when UTI is preadministered prior to LPS stimulation, and serum TNF-a levels are inversely proportional to UTI doses. Other studies have found that ulinastatin does not have a significant anti-inflammatory effect in some experimental models.

In this study, compared with the blank group, serum LEVELS of TNF-a, IL-1 $\beta$ and IL-18 were significantly increased in the diabetic group, indicating that long-term hyperglycemia activates the body's inflammatory response to a certain extent, and hyperglycemia is an important risk factor for sepsis infection. Diabetes sepsis group of rats in the serum TNF alpha, beta, IL IL - 1-18 relative to the first two have increased significantly, and ulinastatin in rat serum inflammatory index fell after pretreatment, suggesting that sepsis infection significantly increase systemic inflammatory response diabetic rats, and ulinastatin can significantly reduce diabetes sepsis systemic inflammatory reaction.

Oxidative stress response is one of the important mechanisms of lung injury in diabetic sepsis. In previous studies, ulinastatin has been reported to have lung protective effects by inhibiting oxidative stress responses. Gao et al. 's study indicated that ulinastatin can reduce the degree of lung injury in burned rats by increasing the generation of SOD and reducing the generation of MDA in lung tissues [20]. In the sepsis model constructed by CLP, hyperglycemia can significantly increase the levels of oxidative factors in the kidney of rats, such as lipid peroxidation (LPO) and catalase (CAT) levels. Meanwhile, SOD activity in kidney tissue was significantly decreased in CLP group ${ }^{\text {[27]. }}$

In this study, it was also found that compared with blank group, the content of MDA in serum of rats in diabetic group increased, while the activity of SOD decreased. The oxidative stress response in serum of diabetic sepsis group was significantly increased; The oxidative stress response in serum of ulinastatin pretreated rats was significantly reduced. This indicates that sepsis infection significantly aggravates the systemic oxidative stress response of diabetic rats. Ulinastatin can significantly reduce systemic oxidative stress response in diabetic sepsis. In this study, ulinastatin can improve oxidative stress and reduce levels of inflammatory factors.

Long-term chronic hyperglycaemia reduces the level of 2, 3-diphosphoglyceric acid in red blood cells, reduces the oxygen carrying capacity of red blood cells, increases the proportion of glycosylated hemoglobin in hemoglobin, and its affinity for oxygen is higher than that of normal hemoglobin, so that oxygen is not easy to diffuse into tissues; Thickening of capillary basement membrane causes chronic ischemia and hypoxia of pulmonary microvessels. Hypoxia inducible factor $1 \mathrm{a}(\mathrm{HIF-1a})$ is a key factor 
specifically mediating hypoxia response in the regulation of oxygen balance in cells. Most cells express HIF-1a in response to hypoxia. Protein stability of HIF-1a is regulated by intracellular oxygen concentration. In cells with normal oxygen content, hiF- 1 a is degraded by protease immediately after translation and thus is largely undetectable. As a mediator of physiological and pathological responses to hypoxia adaptation, hypoxia-inducible factor- 1 is involved in the transcription and expression of many related genes. In vitro studies have shown that hiF-1 amRNA expression increases and hiF-1 a protein accumulation after acute LPS stimulation [21]. In contrast, long-term and sustained LPS stimulation resulted in endotoxin tolerance and reduced hiF-1 a protein content in monocytes ${ }^{[22]}$. In this study, the expression level of HIF-1a protein in the lung tissue of diabetic rats was increased compared with that in the control group, which was consistent with previous studies. However, after LPS induced acute lung injury, hiF-1 a protein expression level in lung tissue was further increased and hypoxia in lung tissue was aggravated. However, ulinastatin pretreatment can down-regulate hiF-1 a protein level in lung tissue, and improve hypoxia response in lung tissue to a certain extent.

Diabetes is a chronic metabolic disorder, and microangiopathy is the characteristic pathological change of diabetes occurring in vital organs of the whole body. A large number of studies have shown that lung tissue rich in microvessels is also one of the target organs of diabetes damage ${ }^{[23,24]}$. It has been reported that microangiopathy is the basis of diabetic pulmonary complications ${ }^{[25]}$. In this study, the content of EB in group $D$ showed an increasing trend compared with the control group, but there was no significant difference, indicating that hyperglycemia can cause pulmonary microvascular injury to a certain extent. After LPS induction, EB content was significantly increased in experimental rats, which further aggravated microvascular injury. After pretreatment with ulinastatin, EB content was significantly lower than that in the DS group, indicating that ulinastatin can improve pulmonary microvascular injury caused by diabetic sepsis, which is consistent with previous reports ${ }^{[26-28]}$.

\section{Conclusion}

This study demonstrates that ulinastatin can effectively relieve acute lung injury induced by diabetic sepsis in rats, and its mechanism may be related to inhibiting inflammatory response, reducing oxidative stress, regulating hypoxia response pathway and improving pulmonary microvascular permeability.

\section{Declarations}

This study was approved by the animal ethics committee at the Zhongnan Hospital and Research Centre, Hubei, China and performed at animal experimental center of Wuhan University, China. The study was carried out in compliance with the ARRIVE guidelines. All methods were carried out in accordance with relevant guidelines and regulations and all experimental protocols were approved by Zhongnan Hospital and Research Centre, Hubei, China and performed at animal experimental center of Wuhan University, China.

Ethics approval and consent to participate 
All experimental procedures were approved by the Animal Ethics Committee of Wuhan University, China, and were performed in accordance with the National Institutes of Health "Guidelines for the Care and Use of Laboratory Animals".

Consent for Publication

Not applicable

Availability of data and material

The datasets used and analysed during the current study are available from the corresponding author on reasonable request.

\section{Competing interests}

The authors declare that they have no competing interest.

\section{Funding contributions}

None.

\section{Acknowledgements}

None

\section{Authors' contributions}

Hu Chen Yufeng Zou and Lijuan Tang finished most of the work of the experiments. Kai Chen and Yufeng Zou wrote the manuscript. Zhe Jin and Meng-Yun Li was responsible for data management. Kai Chen and Yufeng Zou was responsible for the original design and provided critical revisions. Kai Chen and $\mathrm{Hu}$ Chen carried out the statistical analysis and interpretation. All authors read and approved the final manuscript.

\section{Authors' information}

Chen Kai and Zhe Jin are attending physician in the department of anesthesiology at Zhongnan Hospital of Wuhan University. Meng-Yun Li Yufeng Zou and Lijuan Tang are resident physician in the department of anesthesiology at Zhongnan Hospital of Wuhan University.Hu Chen is associate doctor of shenzhen Baoan District maternal and child health care hospital.

\section{Author details}

${ }^{1}$ Department of Anesthesiology, Zhongnan Hospital of Wuhan University, Wuhan, Hubei 430071, P.R. China. ${ }^{2}$ Department of Anesthesiology, Baoan Maternal and Child Health Hospital, Shenzhen®Guangdong 518133, P.R. China.

\section{References}


1. Abdel-Fattah MM. Nosocomial pneumonia: risk factors, rates and trends. East Mediterr Health J. 2008, 14 (3):546-555.

2. Cohendy R, Brougere A, Cuvillon P. Anaesthesia in the older patient. Curr Opin Clin Nutr Metab Care. 2005, 8 (1): 17-21.

3. Lelevich SV, Lelevich VV, Novokshonov AA. Neurotransmitter mechanisms of morphine withdrawal syndrome. Bull Exp Biol Med. 2009 , 148: 184-187.

4. Nguyen MT, Favelyukis S, Nguyen AK, et al. A subpopulation of macrophages infiltrates hypertrophic adipose tissue and is activated by free fatty acids via Toll-like receptors 2 and 4 and JNK-dependent pathways. J Biol Chem. 2007, 282 (48): 35279-35292.

5. Shi H, Kokoeva MV, Inouye K, et al. TLR4 links innate immunity and fatty acid-induced insulin resistance. J Clin Invest. 2006, 116 (11): 3015-3025.

6. Smith LS, Kajikawa O, Elson G, et al. Effect of Toll-like receptor 4 blockade on pulmonary inflammation caused by Mechanical ventilation and bacterial endotoxin. Experimental lung research. 2008, 34 (5): 225-243.

7. Vaneker M, Heunks LMA, Joosten LA et al. Mechanical Ventilation Induces a Toll/Interleukin-1 Receptor Domain-containing Adapter-inducing Interferon beta- dependent Inflammatory Response in Healthy Mice. Anesthesiology, 2009, 111(4): 836-843

8. Rajadas J, Gonzalez E, Ra H, et al. Self-quenching activatable cox-2-specific multi-modal molecular probes for diagnosis of cancers, inflammation, and immune system disorders: U.S. Patent Application 13/932,085[P]. 2013-7-1.

9. Okajima K. Regulation of inflammatory responses by endothelial cells-understanding the molecular mechanism(s) and its therapeutic application to sepsis[J]. Masui,2008,57(3):311-320.

10. Togbe D, Schnyder-Candrian S, Schnyder B, etal. Toll-like receptor and tumour necrosis factor dependent endotoxin-induced acute lung injury. Int J Exp Pathol. 2007, 88 (6): 38 -391.

11. Solomon RL, Corbit JD. 1974. An opponent-process theory of motivation: 1. Temporal dynamics of affect. Psychol. Rev. 81:119-45

12. Fang Y, Xu P, Gu C, Wang Y, Fu XJ, Yu WR, Yao M. Ulina-statin improves pulmonary function in severe burn-induced acute lung injury by attenuating inflammatory response. $J$

Trauma 2011; 71: 1297-130412

1. Jing H, Yao J, Liu X, Fan H, Zhang F, Li Z, Tian X and Zhou Y:Fish-oil emulsion (omega-3 polyunsaturated fatty acids) attenuates acute lung injury induced by intestinal ischemia-reperfusion through Adenosine 5'-monophosphate-activated protein kinase-sirtuin1 pathway. J Surg Res ,2014,187: 252-261,

2. Chen $\mathrm{H}, \mathrm{He} M Y$, Li YM. Treatment of patients with severe sepsis using ulinastatin and thymosin alpha1: a prospective, randomized, controlled pilot study. Chin Med J (Engl), 2009,122 (8): 883-888. 
3. Vaneker M, Heunks LMA, Joosten LA et al. Mechanical Ventilation Induces a Toll/Interleukin-1 Receptor Domain-containing Adapter-inducing Interferon beta- dependent Inflammatory Response in Healthy Mice. Anesthesiology, 2009, 111(4): 836-843

4. Nguyen MT, Favelyukis S, Nguyen AK, et al. A subpopulation of macrophages infiltrates hypertrophic adipose tissue and is activated by free fatty acids via Toll-like receptors 2 and 4 and JNK-dependent pathways. J Biol Chem. 2007, 282 (48): 35279-35292.

5. Shi H, Kokoeva MV, Inouye K, et al. TLR4 links innate immunity and fatty acid-induced insulin resistance. J Clin Invest. 2006, 116 (11): 3015-3025.

6. K Momosaki , N Ishibashi ,S Yoshida, et al. Effect of Preoperative Administration of Methylpredonisolone and Ulinastatin on Tumor Cell Metastasis after Surgical Stress. Kurume Med J, 2014.

7. Meylan E, Burns K, Hofmann K, Blancheteau V, Martinon F, Kelliher M, Tschopp J. RIP1 is an essential mediator of Toll-like receptor 3-induced NF-KB activation. Nature Immunol. 2004, 5(5): 503-507.

8. M Feng , Y Shu , Y Yang, et al. Ulinastatin attenuates experimental autoimmune encephalomyelitis by enhancing anti-inflammatory responses. Neurochem Int, 2014, 64: 64-72.

9. N Huang , F Wang , Y Wang, et al. Ulinastatin improves survival of septic mice by suppressing inflammatory response and lymphocyte apoptosis. J Surg Res, 2013, 182(2): 296-302.

10. Gao C, Liu Y, Ma L, et al.Protective effects of ulinastatin on pulmonary damage in rats following scald injury. Bums. 2012; 38(7):1027-34.

11. S Frede, U Berchner-Pfannschmidt ,J Fandrey. Regulation of hypoxia-inducible factors during inflammation. Methods Enzymol, 2007, 435: 405-419.

12. S Frede, C Stockmann ,S Winning, et al. Hypoxia-inducible factor (HIF) 1alpha accumulation and HIF target gene expression are impaired after induction of endotoxin tolerance. J Immunol, 2009, 182(10): 6470-6476.

13. C Peyssonnaux , P Cejudo-Martin ,A Doedens, et al. Cutting edge: Essential role of hypoxia inducible factor-1alpha in development of lipopolysaccharide-induced sepsis. J Immunol, 2007, 178(12): 75167519.

14. LG Fine ,JT Norman. Chronic hypoxia as a mechanism of progression of chronic kidney diseases: from hypothesis to novel therapeutics. Kidney Int, 2008, 74(7): 867-872.

15. X Xu , P Chen ,Q Zheng, et al. Effect of pioglitazone on diabetic nephropathy and expression of HIF1alpha and VEGF in the renal tissues of type 2 diabetic rats. Diabetes Res Clin Pract, 2011, 93(1): 6369.

16. Feng G, Sun B and Li TZ: Daidzein attenuates lipopolysaccharide-induced acute lung injury via tolllike receptor 4/NF-kappaB pathway. Int Immunopharmacol 2015, 26: 392-400,.

\section{Tables}

Table 1 Comparison of W/D in lung tissues of each group $(\bar{x} \pm s, \mathbf{n}=8)$ 


\begin{tabular}{|lcccl|}
\hline Groups & Group C & Group D & Group DS & Group U \\
\hline W/D & $4.13 \pm 0.19$ & $4.45 \pm 0.13$ & $6.23 \pm 0.45^{\Delta^{*}}$ & $5.31 \pm 0.26^{\Delta^{*}}$ \\
\hline
\end{tabular}

Table 2 Comparison of serum IL-1 $\beta$, IL-18 and TNF-A contents in each group $(\mathrm{pg} / \mathrm{ml}, \mathrm{n}=8, \bar{x} \pm s)$

\begin{tabular}{|lllll|}
\hline Groups & Group C & Group D & Group DS & Group U \\
IL-1 $\beta$ & $62.7 \pm 16.5$ & $153.6 \pm 51.4^{\mathrm{a}}$ & $357.3 \pm 68.1^{\mathrm{ac}}$ & $215.2 \pm 47.3^{\mathrm{abd}}$ \\
IL-18 & $39.8 \pm 10.7$ & $215.9 \pm 62.7^{\mathrm{a}}$ & $571.3 \pm 71.2^{\mathrm{ac}}$ & $427.6 \pm 81.8^{\mathrm{acd}}$ \\
TNF-a & $21.5 \pm 5.6$ & $311.7 \pm 33.8^{\mathrm{a}}$ & $639.1 \pm 52.0^{\mathrm{ac}}$ & $389.2 \pm 36.2^{\text {abe }}$ \\
\hline
\end{tabular}

Note: Compared with group $C$, ${ }^{a} P<0.01$; Compared with group $D,{ }^{b} P<0.05,{ }^{c} P<0.01$; Compared with $D S$ group, ${ }^{\mathrm{d}} P<0.05,{ }^{\mathrm{e}} P<0.01$

Table 3 Comparison of serum MDA and SOD contents in each group $(\mathbf{n}=8, \bar{x} \pm s)$

\begin{tabular}{|lll|}
\hline Groups & MDA contents $\triangle \mathrm{nmol} / \mathrm{ml} \mathbb{}$ & SOD activity $\triangle \mathrm{U} / \mathrm{ml}$ \\
\hline Group C & $5.1 \pm 0.32$ & $132.4 \pm 20.8$ \\
Group D & $8.8 \pm 1.19^{\mathrm{a}}$ & $91.3 \pm 16.4^{\mathrm{a}}$ \\
Group DS & $20.6 \pm 3.25^{\mathrm{ac}}$ & $56.3 \pm 12.1^{\mathrm{ac}}$ \\
Group U & $10.5 \pm 2.04^{\mathrm{abd}}$ & $93.6 \pm 12.5^{\mathrm{ad}}$ \\
\hline
\end{tabular}

Note: Compared with group $C,{ }^{a} P<0.01$; Compared with group $D,{ }^{b} P<0.05,{ }^{c} P<0.01$; Compared with DS group, ${ }^{\mathrm{d}} P<0.01$

Table 4 Comparison of TLR4 mRNA expression in lung tissues of rats in each group $\bar{x} \pm s, \mathrm{n}=8[$

\begin{tabular}{|lllll|}
\hline Groups & Group C & Group D & Group DS & Group U \\
\hline${ }^{2-\Delta \Delta}$ Ct Vaule & $1.00 \pm 0.11$ & $1.63 \pm 0.22^{\Delta}$ & $6.90 \pm 1.01^{\Delta^{*}}$ & $2.68 \pm 0.41^{\Delta^{* \#}}$ \\
\hline
\end{tabular}

Note: Compared with group $C,{ }^{\Delta} P<0.01$; Compared with group $D,{ }^{*} P<0.01$; Compared with DS group, ${ }^{\#} P<0.01$

Table 5 Comparison of EB content in lung tissues of rats in each group $\bar{x} \pm s, \mathbf{n}=4 \square$

\begin{tabular}{|lllll|}
\hline Groups & Group C & Group D & Group DS & Group U \\
\hline EB囚ug/mg $\$ & $31.8 \pm 3.9$ & $36.2 \pm 2.5$ & $63.6 \pm 7.3^{\Delta^{*}}$ & $52.1 \pm 4.7^{\Delta^{*}}$ \\
\hline
\end{tabular}


Note: Compared with group $C,{ }^{\triangle} P<0.01$; Compared with group $D$, ${ }^{*} P<0.01$; Compared with DS group, ${ }^{\#} P<0.01$

\section{Figures}
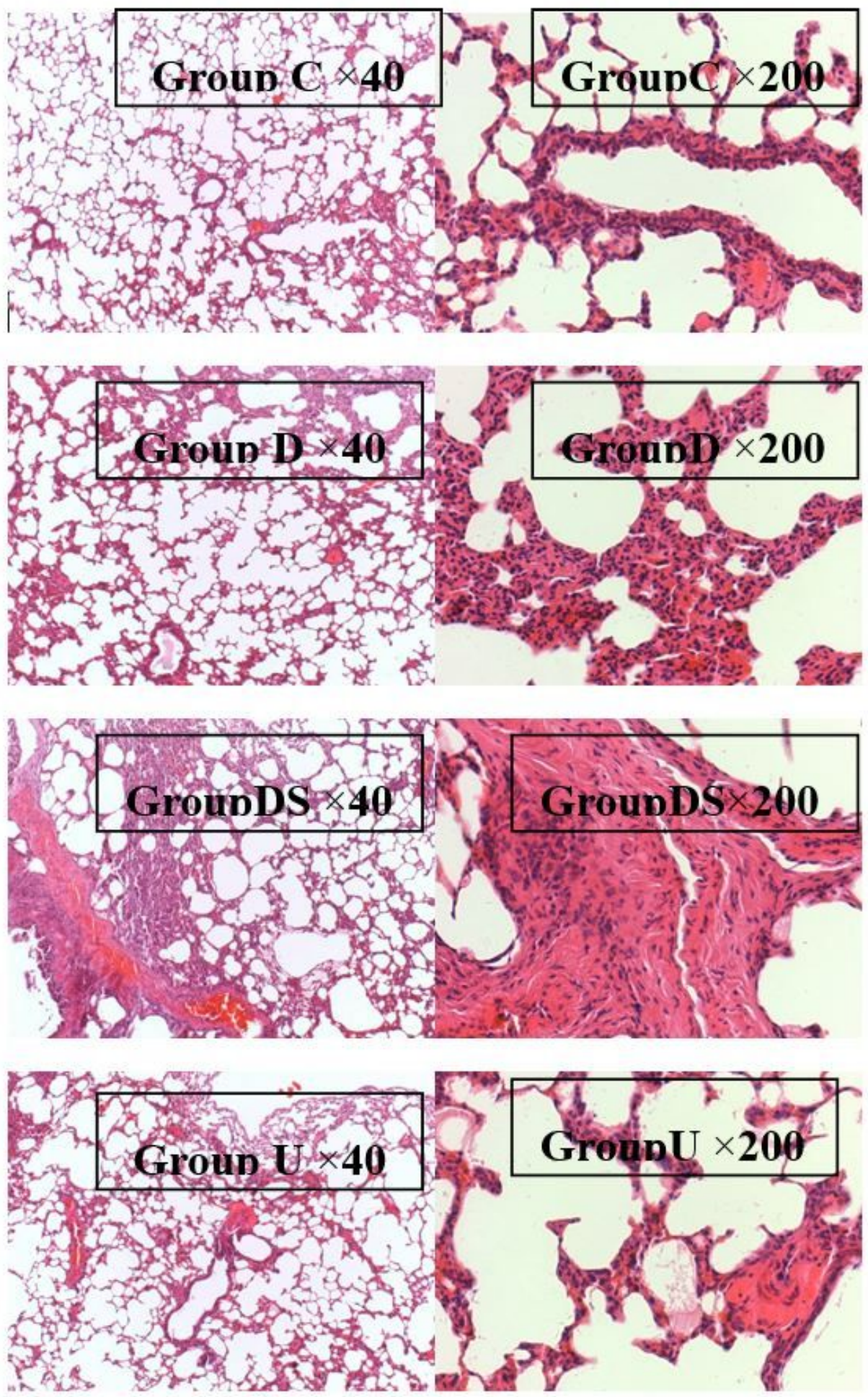

Figure 1 
HE staining of lung tissues of rats in each group $₫ n=8 \rrbracket$
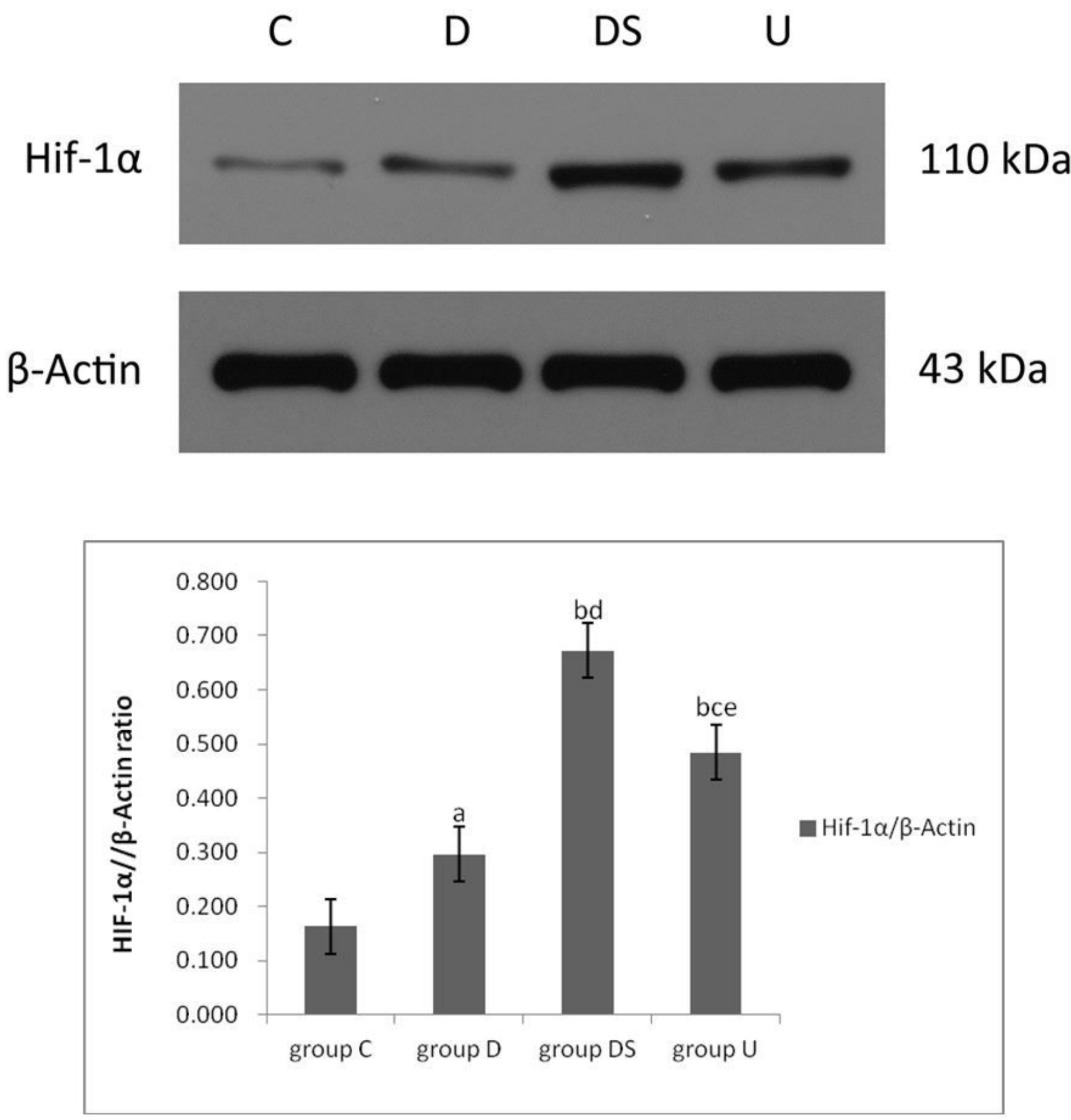

Figure 2

Expression of hypoxia-inducible factor-1 a protein in kidney tissue detected by Western blot Note: Compared with group $\mathrm{C}, \mathrm{aP}<0.05, \mathrm{bP}<0.01$; Compared with group $\mathrm{D}, \mathrm{cP}<0.05, \mathrm{dP}<0.01$; Compared with $\mathrm{DS}$ group, $\mathrm{eP}<0.05 \llbracket \mathrm{n}=8 \rrbracket$ 